METABOLISM

\section{AMP is the champ}

Cell Metab. 18, 556-566 (2013)

AMP-activated protein kinase (AMPK) senses falling cellular energy status and activates catabolic pathways while inhibiting anabolic pathways to restore energy homeostasis. AMPK is activated by the upstream kinases LKB1 and CAMKK- $\beta$, which phosphorylate Thr172. The nucleotides ADP and AMP (signals of low cellular energy) promote Thr172 phosphorylation, whereas AMP causes further allosteric activation. Recently, ADP had been proposed to be the primary physiological signal, as it is present at higher cellular concentrations than AMP. However, Gowans et al. found that AMP was tenfold more potent than ADP in inhibiting Thr172 dephosphorylation, whereas it was the sole enhancer of Thr172 phosphorylation by LKB1. They also found the effect of allosteric activation was larger (greater than tenfold), whereas the increase in Thr172 phosphorylation in intact cells was smaller (less than fourfold) than previously thought. The authors further examined the role of allosteric activation in intact cells. They administered two compounds: the metabolic inhibitor berberine, which increases AMP, and another allosteric activator, A769662, under conditions where Thr172 phosphorylation could not occur. Both compounds still markedly stimulated the phosphorylation of downstream targets, showing that allosteric activation is an important mechanism. These studies suggest that AMP is the primary signal activating the AMPK system and stress the importance of the allosteric mechanism.

\section{PROTEIN DYNAMICS}

\section{Pomp and SERCAmstance \\ Proc. Natl. Acad. Sci. USA,}

doi:10.1073/pnas.1303006110
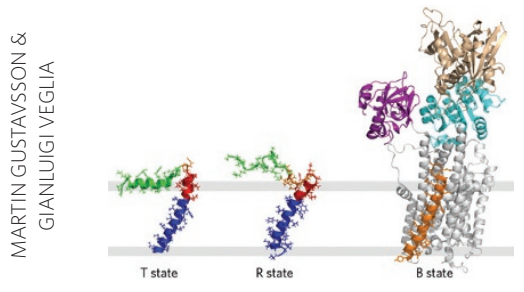

The contraction of muscle cells occurs when $\mathrm{Ca}^{2+}$ ions are released from the sarcoplasmic reticulum and ceases when those ions are resequestered by the sarcoplasmic reticulum $\mathrm{Ca}^{2+}$-ATPase (SERCA). Phospholamban (PLN), a small integral membrane protein, inhibits SERCA activity in vivo; the phosphorylation of PLN by protein kinase A alleviates SERCA inhibition. Gustavsson et al. now report that the conformational dynamics of PLN have an important role in this process. In the absence of SERCA, the regulatory Ia domain of PLN exists in two major conformational states: a helical, membrane-associated ' $\mathrm{T}$ ' state and a disordered, membrane-unassociated ' $R$ ' state. The authors used solid-state NMR spectroscopy to show that phosphorylation of PLN shifts the equilibrium from the $\mathrm{R}$ state toward a third, SERCA-bound ('B') state, which is sparsely populated when PLN is not phosphorylated. Although the large size of the phospho-PLN-SERCA complex $(\sim 116 \mathrm{kDa})$ prevented the authors from using NMR spectroscopy to determine its atomic structure, they were able to show that the phosphate group of phospho-PLN binds an allosteric site between SERCA's nucleotide-binding and phosphorylation domains. Additional work is needed to determine exactly how the structure of the active phospho-PLN-SERCA complex differs from that of the inactive PLN-SERCA complex.

$J M F$

\section{REACTION DISCOVERY}

\section{Now we're clicking}

Angew. Chem. Int. Ed. Engl., doi:10.1002/ anie. 201305645

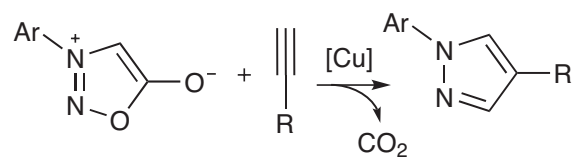

The discovery of new chemical reactions can be driven by serendipity, rational design or-increasingly-screening. Recent research reported the use of sandwich immunoassays as a method to identify coupling of fragments attached to antibody tags. Kolodych et al. now employ this method to search for new $[3+2]$ cycloaddition reactions akin to the well-known Huisgen cycloaddition, or azide-alkyne click reaction. The authors screened 11 dipoles, 8 'dipolarophiles' and 31 transition metal catalysts in 96-well plates; of the nearly 3,000 reactions tested, 51 yielded hits, and 42 of these were new. As the initial yields were low, the authors further optimized the reactions by screening different reaction conditions; once improved, the reactions were analyzed to determine their efficiency, chemoselectivity and cross-reactivity with biological samples. Four reactions reached yields of more than $50 \%$ after optimization, and one of these-the coupling of arylsydnones with alkynes-is a known reaction but has always required harsh conditions to yield limited product. The reaction screening performed here identified copper phenanthroline complexes as catalysts able to reach up to $99 \%$ yield and capable of converting a variety of substrates, even in the presence of cell culture media. This ' $\mathrm{Cu}$-catalyzed sydnone-alkyne cycloaddition' (CuSAC) is a welcome addition to the chemical biologist's toolbox.

$C G$

\section{METAL REGULATION}

\section{SOD1 off zinc}

\section{Mol. Cell 52, 75-86 (2013)}

Pathogenic versions of the metalloenzyme copper zinc superoxide dismutase (SOD1) have been detected in the neurodegenerative disorder amyotrophic lateral sclerosis. Recent studies have suggested that mutant forms of SOD1 might contribute to ER stress and subsequent toxicity via interactions with the ER-associated degradation membrane protein derlin-1. Surprisingly, the wild-type form of SOD1 contains a hidden derlin-1 binding site (DBS) that mediates binding under serumstarved conditions. The reintroduction of serum blocks this interaction, but the serum component that modulates SOD1-derlin-1 interactions remained unclear. Homma et al. performed gel filtration chromatography of fetal bovine serum (FBS) fractions to identify the serum component, which was revealed to be inorganic. The authors found that the addition of zinc could disrupt SOD1-derlin-1 interactions through the concealing of the DBS site. Reduction in zinc levels through addition of chelators such as TPEN provoked changes in SOD1 conformation through exposing the DBS, resulting in the upregulation of the ER stress machinery. Finally, activation of the ER stress components PERK and ATF6 decreased protein synthesis and induced the expression of the zinc transporter, ZIP14, respectively. Thus, the SOD1-derlin-1 complex provides a critical link between the cellular responses to ER stress and zinc deficiency. 